\title{
A simulation model of a floating half zone
}

\author{
Z.M. Tang*, W.R. Hu \\ Institute of Mechanics, CAS, Beijing 100080, People's Republic of China \\ National Microgravity Laboratory, CAS, Beijing 100080, People's Republic of China \\ Received 9 January 1998; accepted 23 February 1998
}

\begin{abstract}
A simulation model of a floating half zone was suggested by steady numerical simulation and experiment respectively, in the previous papers [Q.S. Chen, W.R. Hu, Int. J. Mass Heat Transfer 40 (1997) 757; J.H. Han, Y. Ar, R. Zhou, W.R. Hue, Int. J. Mass Heat Transfer 40 (1997) 2671]. In the present paper, the simulation model is studied by using the method of unsteady and three-dimensional numerical simulation, and the transient process from steady convection to oscillatory convection is especially analyzed. Comparison of onsets of oscillation for both simulation model and the usual model were obtained, and the results show that the critical Marangoni number of the simulation model is obviously smaller than that of the usual model for the same slender liquid bridge. This implies that the usual model of a floating half zone gives a lower estimation on the onset of oscillation for floating zone convection. (C) 1998 Elsevier Science B.V. All rights reserved.
\end{abstract}

Keywords: Thermocapillary convection; Onset of oscillation; Floating half zone; Numerical simulation

\section{Introduction}

The floating zone technique is used for the growth of high-quality crystal especially in the microgravity environment, where thermocapillary convection dominates the process in comparison with the buoyancy convection. To simulate the thermocapillary convection in the floating zone process, a model of a floating half zone was suggested by Chang and Wilcox in 1975 [3] as shown in

* Corresponding author.
Fig. 1a, where a liquid bridge was floated between two co-axial rods with constant temperatures, and this model is named as the usual model of a floating half zone. The onset of oscillation in thermocapillary convection is most interesting due to its importance in both the research of hydrodynamic instability [4] and the application of materials processings [5-8]. The thermocapillary effect on the ground becomes relatively important in comparison with the buoyancy effect if the typical scale of the liquid bridge is decreased and a small floating half zone on the ground is extensively studied to simulate the thermocapillary process in a floating 

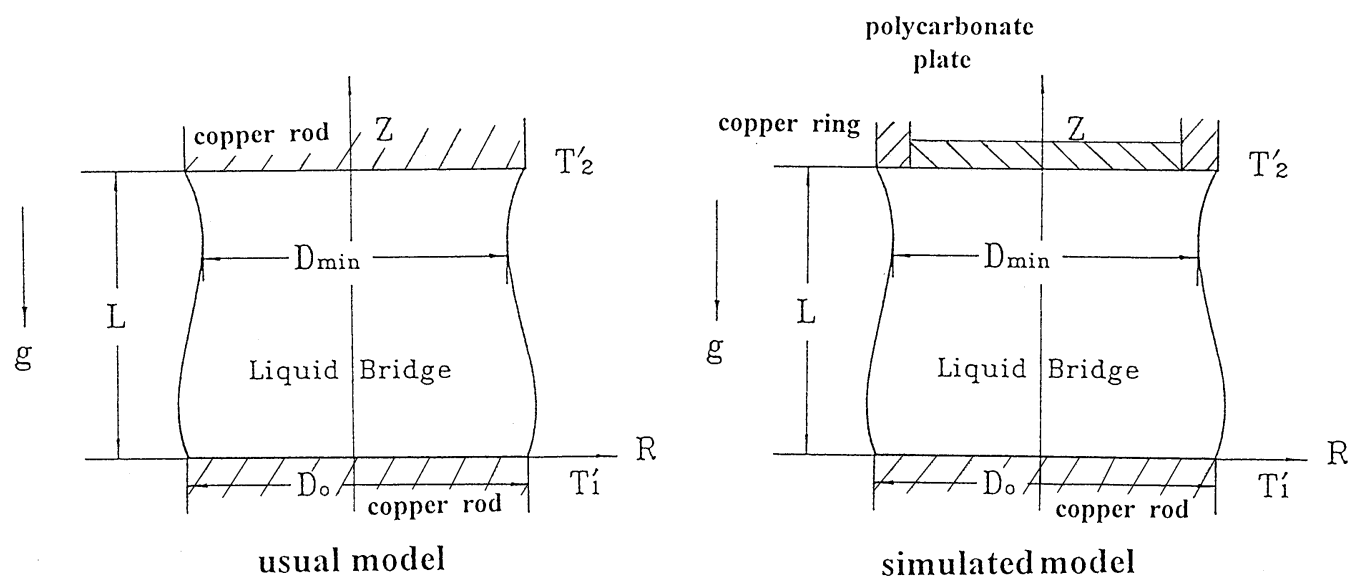

Fig. 1. Schematic diagram of the liquid bridge.

zone in a microgravity environment in the last two decades.

The usual model of floating half zone is a simplified hydrodynamic model and is better for studies of the thermocapillary effect. The temperature and flow field is symmetric to the middle plane of a full floating zone in the microgravity environment if the effect of the pulling velocity of the crystal growth is neglected. It can be seen that the thermal condition at the middle plane is not a constant temperature, but gives a temperature distribution with positive radial gradient; that is, the temperature is highest at the free surface of the middle plane and decreases to the center. A modified model of a floating half zone was suggested for observing the velocity of threedimensional distribution. The liquid bridge is floated between one copper rod and a disk made of transparent materials surrounded by ring heater in a configuration similar to Fig. 1a. The thermal boundary conditions are a constant temperature at the copper boundary and a temperature distribution at the disk boundary. Sapphire $[9,10]$ and glass [11] are selected as the disk materials. Because of the heat conductivity of sapphire or glass is at least one order of magnitude larger than that of an experimental liquid such as silicon oil, the temperature distribution at the disk boundary gives a negative radial gradient, which do not agree with the requirement at the middle plane of a full floating zone [1,2]. In addition, there is only a small radial temperature gradient at the disk boundary
$[2,12]$, and the modified model is nearly the same as the usual model of a floating half zone.

A simulation model of a floating half zone as shown in Fig. $1 \mathrm{~b}$ was suggested to simulate the thermal condition at the middle plane of a full floating zone, and the disk of transparent materials consists of polycarbonate, which has a thermal conductivity larger than the experimental liquid of silicon oil and gives a large and positive radial temperature gradient $[1,2]$. The fundamental features were studied using numerical analyses for steady convection [1] and the experimental method for both steady and oscillatory convection [2]. In the present paper, the transient process from steady convection to oscillatory convection is studied numerically for the simulation model, and an unsteady and three-dimensional program is applied to analyze the onset of oscillation. The critical Marangoni number of the simulation model is compared with that of the usual model, and the results show that the critical Marangoni number of the usual model is obviously larger than that of the simulation model for a liquid bridge with a suitable volume.

\section{Description of the model}

The equations and boundary conditions for a simulation model as shown in Fig. 1b could be described as follows [12]. 
Equations. The nondimensional parameters, the Reynolds number Rs, Marangoni number Ma, and Grashof number $\mathrm{Gr}$ are introduced as

$\mathrm{Rs}=\frac{U_{r}^{\prime} L}{v}, \quad \mathrm{Ma}=\frac{U_{r}^{\prime} L}{\kappa}, \quad \mathrm{Gr}=\frac{g \beta\left(T_{2}^{\prime}-T_{1}^{\prime}\right) L^{3}}{v^{2}}$,

where $\beta, v, \kappa$, are the thermal expansion coefficient, kinematic viscosity, and thermal diffusion coefficient, respectively; $g$ is the gravity, and $U_{*}^{\prime}=|\partial \sigma / \partial T|\left(T_{2}^{\prime}-T_{1}^{\prime}\right) / \rho v$ is the typical velocity, and superscript prime denotes a dimensional quantity. The nondimensional unsteady and threedimensional equations for vector stream function $\psi$, vector vorticity $\omega$, and temperature $T$ in the cylindrical coordinate system $(r, \theta, z)$ may be written as

$\nabla \times \nabla \times \psi=\omega$,

$\frac{\partial \omega}{\partial t}+\boldsymbol{V} \cdot \nabla \omega-\omega \cdot \nabla \boldsymbol{V}=\frac{1}{\mathrm{Rs}}\left(\nabla^{2} \boldsymbol{\omega}+\nabla \times \boldsymbol{F}\right)$,

$\frac{\partial T}{\partial t}+\boldsymbol{V} \cdot \nabla T=\frac{1}{\mathrm{Ma}} \nabla^{2} T$,

the $\psi$, and $\omega$ related to velocity $\boldsymbol{V}$ are defined by

$\nabla \times \psi=V, \quad \nabla \times V=\omega$.

External force $\boldsymbol{F}$ is considered as gravitational force only. In the above formula, the scale, velocity, and temperature are nondimensional, respectively; the height of liquid bridge $L$, typical velocity $U_{*}^{\prime}=|\partial \sigma / \partial T| \Delta T / \rho v$, and $\Delta T$, where $\sigma, \rho$ and $\Delta T$ are, respectively, the surface tension, density of liquid, and applied temperature difference between the copper rod and the heater around the disk.

Boundary conditions. The boundary conditions are as follows:

$\psi_{r}=\psi_{\theta}=0, \quad \frac{\partial \psi_{z}}{\partial z}=0$,

$\omega_{r}=-\frac{\partial v}{\partial z}, \quad \omega_{\theta}=\frac{\partial u}{\partial z}, \quad \omega_{z}=0$

$T(r, \theta, 0, t)=0, \quad T(r, \theta, 1, t)=1$,

for the usual model $\frac{\partial T(r, \theta, 1, t)}{\partial z}=0, \quad 0 \leqslant r \leqslant R(1)-\delta$,

$T(r, \theta, 1, t)=1, \quad R(1)-\delta \leqslant r \leqslant R(1)$

for simulation model

at the liquid-solid interface, and

$$
\begin{aligned}
\psi_{s}= & \psi_{\theta}=0, \quad \nabla \cdot \psi=0 \\
\omega_{\phi}= & -\left\{\frac{\left(1+f^{\prime 2}\right)}{\left(1-f^{\prime 2}\right)} \frac{\partial T}{\partial S}\right. \\
& \left.+\frac{2 f^{\prime}}{\left(1-f^{\prime 2}\right)}\left(\frac{\partial u}{\partial r}-\frac{\partial w}{\partial z}\right)+2 \frac{\partial u}{\partial z}\right\}, \\
\omega_{z}= & \frac{\left(1+f^{\prime 2}\right)^{1 / 2}}{r} \frac{\partial T}{\partial \phi}+2 \frac{\partial v}{\partial r}-f^{\prime}\left(\omega_{r}+2 \frac{\partial v}{\partial z}\right), \\
\omega_{r}= & \frac{1}{r} \frac{\partial w}{\partial \phi}-\frac{\partial v}{\partial z}, \\
\frac{\partial T}{\partial n}= & 0,
\end{aligned}
$$

at the free surface of the liquid, where $r=f(z)$ is the free surface equation, the superscript prime of $f$ means the differentiation of $f$ along $Z$ coordinate, $n$ denotes normal coordinate, and $\delta$ is the difference between external and internal radii of the upper copper ring.

Initial conditions. At initial time, the applied temperature difference $\Delta T$ is zero, the velocity is zero, and the temperature is uniform in the liquid bridge. Then, the applied temperature difference is gradually increased by a step of $2^{\circ} \mathrm{C} / \mathrm{s}$ to continue the calculation. As the applied temperature difference increased with time, typical velocity will also depend on time. Therefore, a nondimensional time is adopted as

$t=\int_{0}^{t^{\prime}}\left(\frac{U_{*}^{\prime}}{L}\right) \mathrm{d} t^{\prime}$

in the present calculation.

Numerical calculation. The finite element method is used in the calculation. The floating half zone is divided into 16416 tetrahedron elements with 3128 nodes, and the number of grids are 9, 24, 17 in the direction $r, \theta, z$, respectively. In order to calculate conveniently the nonlinear effects of convections, 
Table 1

Physical properties of $10 \mathrm{cSt}$ silicon oil

\begin{tabular}{lllll}
\hline Fluid & $\rho\left(\mathrm{kg} / \mathrm{m}^{3}\right)$ & $v\left(\mathrm{~m}^{2} / \mathrm{s}\right)$ & $\kappa\left(\mathrm{m}^{2} / \mathrm{s}\right)$ & $\beta(1 / \mathrm{K})$ \\
\hline Silicon oil $10 \mathrm{cSt}$ & $9.35 \times 10^{2}$ & $1 \times 10^{-5}$ & $9.47 \times 10^{-8}$ & $1.08 \times 10^{-3}$ \\
\hline
\end{tabular}

the vorticity equation and the energy equation are separated into the convection part and the diffusion part, which are, respectively, dealt with by the characteristic line method and the finite element method. The calculated results agree with other publications, and the details are discussed elsewhere.

Computational model. An example is analyzed numerically in detail in the present paper. The $10 \mathrm{cSt}$ silicon oil is adopted as typical medium. The physical properties of $10 \mathrm{cSt}$ silicon oil are shown in Table 1. The geometrical aspect ratio, that is, the ratio of the height of the liquid bridge to the diameter of the copper rod, is 0.7 for a liquid bridge of height $3.5 \mathrm{~mm}$. The volume of the liquid bridge is 0.805 the volume of a cylinder with the same height and aspect ratio. The copper rod is on the lower side and the disk surrounding a heater is on the upper side. The gravity on the earth condition is considered for comparison with the ground-based experiment.

\section{Transient process}

Thermocapillary convection is steady if the applied temperature difference is smaller than a critical value, and the velocity field and temperature distribution are axi-symmetric. The steady convection of the simulation model was studied in Ref. [1], and the onset of oscillation and the oscillatory convection will be discussed here.

A typical evolution process of the simulation model is studied numerically for a small liquid bridge of height $3.5 \mathrm{~mm}$ and of radius $2.5 \mathrm{~mm}$ of disk and copper rod, and the volume of liquid bridge is 0.805 of the volume of the cylinder. The evolution curves at four rectangular positions at the free surface in a cross-section $z / l=0.5$, and the evolutions of azimuthal velocity components and temperature profiles are given, respectively, in Fig. 2a and Fig. 2b. The evolution curves at four positions of the same radius are overlapped and changed slowly for steady convection, and are oscillated and split with different phases. The onset of oscillation is in a period but not at a moment as discussed experimentally in Ref. [13]. The start of the period is marked by splitting the evolution curve at different positions of same radius in a horizontal cross-section, and the completion of the period may be defined by the moment on the completion of the oscillation.

The oscillatory feature may be described by the axial asymmetry of the typical quantities, which may be defined nondimensionally, for example, as follows:

$N_{\mathrm{v}}=\left(V_{\max }-V_{\min }\right) / V^{*}$,

where the subscript max and min denote, respectively, the maximum and minimum value of azimuthal velocity components on a circle of free surface in the horizontal cross-section $z / L=0.5$, and the typical velocity $V^{*}$ is adopted as the maximum velocity in the liquid bridge. The evolutions of typical quantity $N_{\mathrm{v}}$ at three horizontal crosssections are shown in Fig. 3 for the simulation mode. The maximum asymmetry appears near the plane $z / L=0.5$, and then decreases on both sides. The asymmetry is larger in the cross-section, for example $z / L=0.26$, of the lower side close to the copper rod in comparison with the cross-section, for example $z / L=0.74$, of the upper side close to the transparent disk. The onset of oscillation described by the oscillatory feature, may be described by the axial asymmetric quantity $N_{\mathrm{v}}$, as shown in Fig. 4 for both the simulation model and the usual model. It can be seen clearly at the period of the onset of oscillation, and the critical Marangoni 

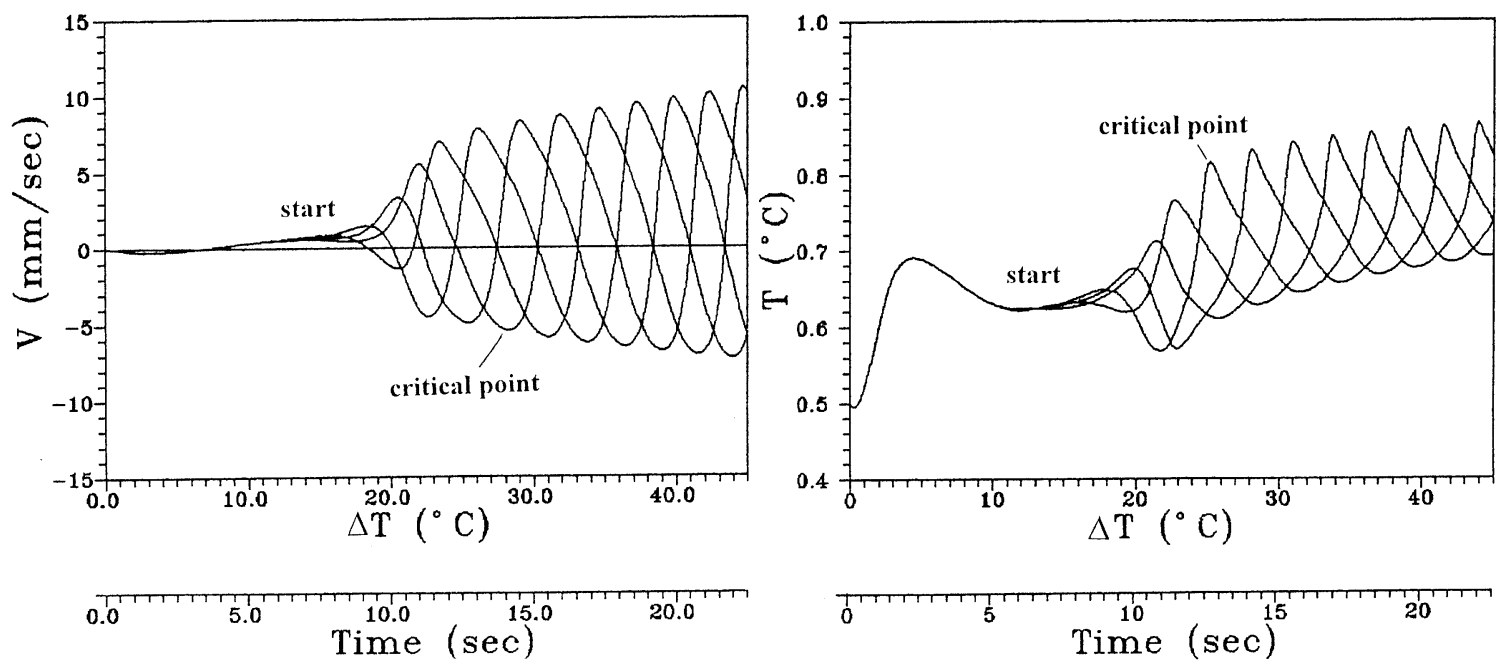

Fig. 2. The evolution process of azimuthal velocity $V$ (left) and temperature (right) at four spots $\varphi=0, \pi / 2, \pi, 3 \pi / 2$ of the free boundary $z / L=0.5$.

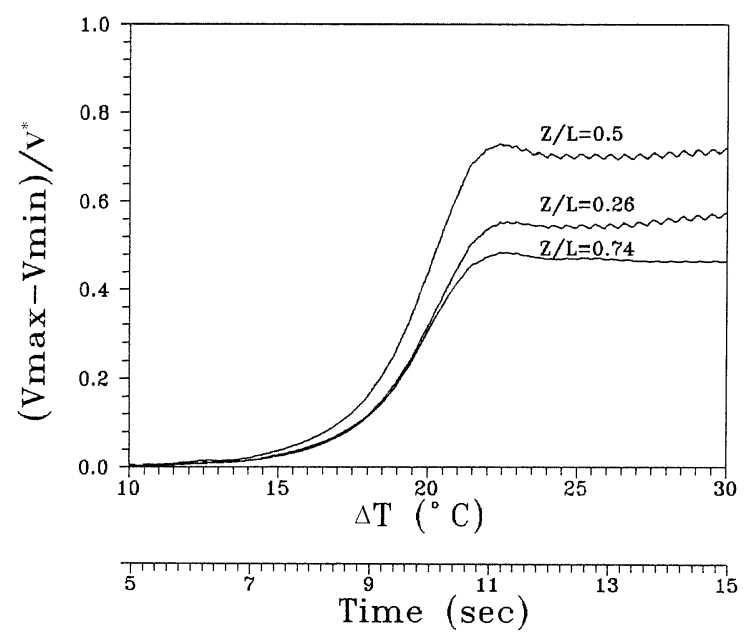

Fig. 3. The relationship between $N_{\mathrm{v}}=\left\{\left(V_{\max }-V_{\min }\right) / V^{*}\right\}$ and $\Delta T$ at boundaries of horizontal cross-section $z / L=0.26,0.5$, 0.74 .

number of the simulation mode is only $81 \%$ of that of the usual model. This conclusion implies that the thermal boundary condition is sensitive to the onset of oscillation in the half floating zone convection, and the usual model of the half floating zone gives a lower estimation on the danger of the onset of oscillation. This conclusion agrees with the groundbased experiment [2].

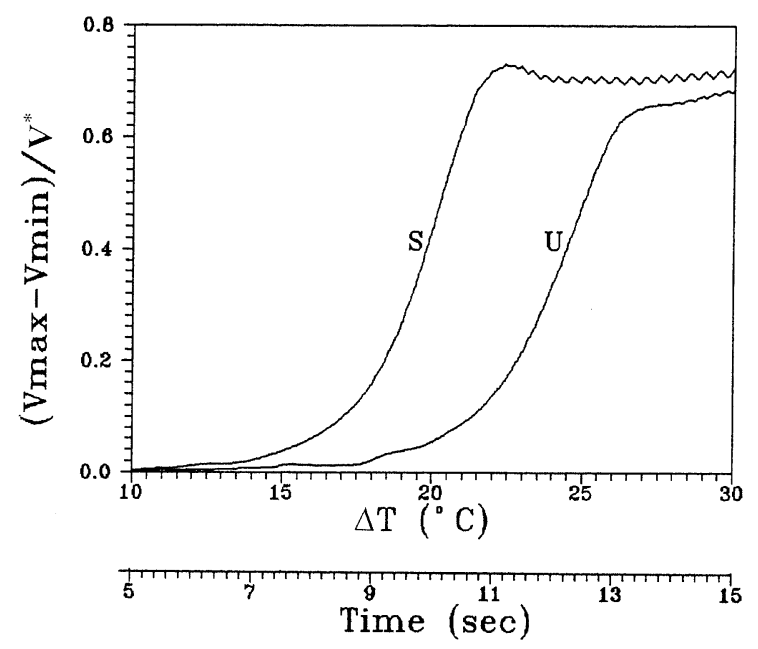

Fig. 4. The comparison of $N_{\mathrm{v}}$ for simulated and usual models. $(z / L=0.5)$.

Temperature profiles and velocity distributions of the azimuthal component in one period are given in Fig. 5, which presents clearly that the pattern of temperature profile rotates in a frequency as the same as other quantities; however, the fluid does not rotate with the same frequency as shown by the oscillatory azimuthal velocity. The same conclusion was obtained by both numerical and 

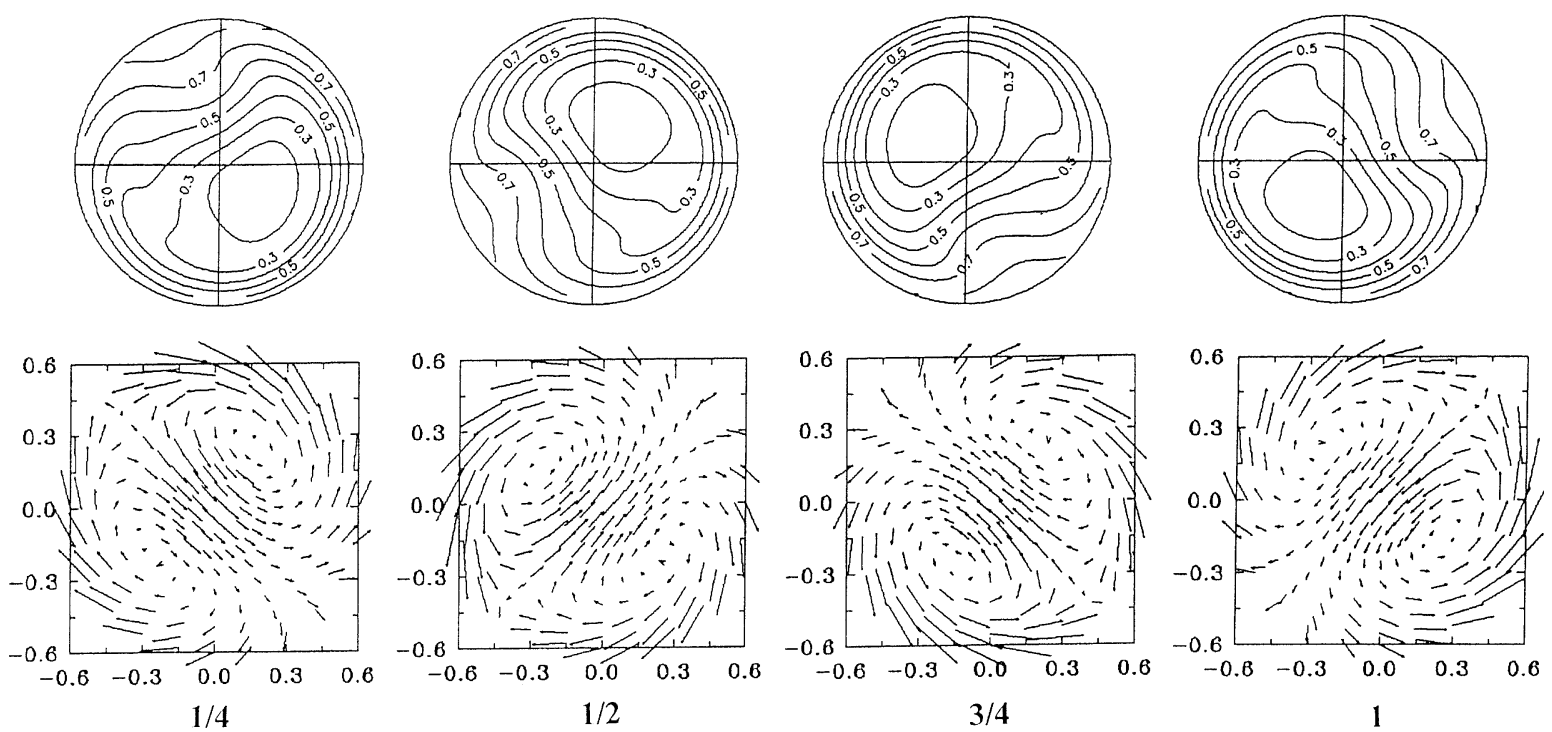

Fig. 5. Flow fields (lower) and temperature distribution (upper) in a sequence $\frac{1}{4}, \frac{1}{2}, \frac{3}{4}, 1$ period, at $z / L=0.5, \Delta T=45^{\circ} \mathrm{C}$ (simulated model).

experimental simulations for the usual model $[12,14]$, and these conclusions disagree with those of Ref. [11], in which authors suggested that the fluid rotates together with the pattern. It can be estimated that the fluid rotation with the frequency needs a large amount of kinematic energy, which is not easy to be supported.

\section{Conclusions}

The simulation model is discussed by using the three-dimensional and unsteady numerical method in the present paper, and the features of the transient process are analyzed for a fixed liquid bridge. The results show that the thermal boundary condition at the solid surface is sensitive to the internal fields, and the thermal boundary condition of the simulation model gives better results for simulating half of a full floating zone in comparison with the usual model. The critical Marangoni number of the simulation model is obviously smaller than that of the usual model for a volume of liquid bridge discussed in the present paper, and it means that the onset of oscillation is more easy to be excited in the simulation model.
The evolution of temperature and velocity distributions were also obtained. The results show that the pattern of temperature profile rotates with the same frequency as that of velocity patterns but the velocity is oscillatory in a horizontal cross-section for the oscillatory convection of both the simulation model and the usual model. This conclusion agrees with our experiment and numerical simulation [2,12], and also with 3-D numerical results in the paper of Savino and Monti [15], but disagrees with the suggestion of Ref. [11].

The thermal boundary condition at one solid boundary is improved in the simulation model. However, the velocity boundary conditions at both solid boundaries are zero for both the simulation model and usual model, but there is a radial velocity component in the middle plane of a full floating zone. This implies that the velocity boundary conditions of both the simulation model and the usual model at the heater's plane are different from the one in the middle plane of a full floating zone.

The features of transient process are analyzed for a simulation model of the floating half zone, and more samples should be discussed in the next step. 


\section{Acknowledgements}

The project is supported partly by the National Natural Science Foundation (19789201) of China.

\section{References}

[1] Q.S. Chen, W.R. Hu, Int. J. Mass Heat Transfer 40 (1997) 757.

[2] J.H. Han, Y. Ar, R. Zhou, W.R. Hue, Int. J. Mass Heat Transfer 40 (1997) 2671.

[3] C.E. Change, W.R. Wilcox, J. Crystal Growth 28 (1975) 8.

[4] M.K. Smith, S.H. Davis, J. Fluid Mech. 132 (1983) 119.

[5] C.-H. Chun, J. Crystal Growth 48 (1980) 600.

[6] Y. Kamotani, S. Ostrach, M. Vargas, J. Crystal Growth 66 (1984) 83.
[7] D. Schwabe, A. Scharmann, J. Crystal Growth 46 (1979) 124.

[8] W.R. Hu, J.Z. She, R. Shoo, Z.M. Tang, J. Crystal Growth 142 (1994) 379.

[9] A. Whereat, S. Nishizawa, M. Sakurai, Jpn. Soc. Microgr. Appl. 11 (1992) 189.

[10] A. Hirata, S. Nishizawa, M. Noguchi, M. Sacra, S. Yasuhiro, N. Imaishi, J. Chem. Eng. Japan 27 (1994) 65.

[11] J. Lee, D.J. Lee, J.H. Lee, J. Crystal Growth 152 (1995) 341.

[12] Z.M. Tang, W.R. Hu, G. Chen, B. Roux, 47th Int. Astronautical Congress, Oct. 7-11, 1996, Beijing, China. IAF96-J.4.02.

[13] Y.L. Yao, F. Liu, W.R. Hu, Int. J. Mass Heat Transfer 39 (1995) 2539.

[14] Y. Ar, Z.M. Tang, W.R. Hu, Microgr. Sci. Appl. 10 (1997), in press.

[15] R. Savino, R. Monti, Phys. Fluids 8 (1996) 2096. 